\title{
Characterizing the Effects of Radiation Dermatitis on Quality of Life
}

Alexandra Rzepecki BS ${ }^{1,2}$, Mathew Birnbaum MD², Nitin Ohri MD², Johanna Daily MD, Jana Fox MD³, William Bodner MD³, Rafi Kabarriti MD, Madhur Garg MD³, Keyur Mehta MD³, Shalom Kalnicki MD³, Beth McLellan MD²

${ }^{1}$ University of Michigan Medical School. Ann Arbor, MI ${ }^{2}$ Division of Dermatology, Montefiore Medical Center. Bronx, NY ${ }^{3}$ Department of Radiation Oncology, Montefiore Medical Center. Bronx, NY ${ }^{4}$ Division of Infectious Diseases. Albert Einstein COM. Bronx, NY

\section{Background:}

- Acute radiation dermatitis $(\mathrm{RD})$ is one of the most common adverse events of radiation therapy (RT), affecting up to $95 \%$ of patients undergoing treatment

- Acute RD may present with hyperpigmentation, erythema, and dry and moist desquamation

- Little is known about how radiation-induced skin toxicity can affect patients' quality of life (QoL) during treatment

\section{Objective:}

- To quantify the impact of radiation dermatitis on QoL in patients undergoing RT using the Skindex-16, a validated instrument used to assess how a skin disease affects QoL in 3 domains: emotions, symptoms, and functioning

\section{Methods:}

- An IRB-approved prospective study was conducted including subjects undergoing RT for treatment of cancers of the breast, head and neck, and anus at Montefiore Medical Center

- Skindex-16 was administered at 2 time points:

1) Prior to initiation of RT (pre-treatment)

2) Upon completion of RT (post treatment)

- Subjects were evaluated weekly during RT and dermatitis was graded using the Common Terminology Criteria of Adverse Events (CTCAE) v4.03

- Pre- and post- treatment Skindex-16 scores were compared using the sign test

- Spearman rank correlation was used to assess associations between changes in Skindex-16 scores and most severe dermatitis grade

\section{Results:}

90 subjects met inclusion criteria

7 subjects did not finish $R T$

\section{3 subjects completed the Skindex-16} questionnaire pre- and post- radiotherapy
Cancer

Breast, $n=41(49 \%)$

Head \& neck, $n=37(45 \%)$

Anal, $n=5(6 \%)$
Dermatitis grade

Grade 1, n=49 (59\%)

Grade 2, $n=27(33 \%)$

Grade 3, $n=7(8 \%)$

\section{Results:}

- The median composite Skindex-16 score was 0 prior to RT (IQR 0-9) and 34 (IQR 9 to 56) upon RT completion

- Patients reported worsened skin condition for every question after RT compared to before RT (Sign test $p$ values $<0.001$ )

- The greatest degree of worsening was observed for "itching", "burning or stinging" and "being irritated"

- An increase composite Skindex-16 score was associated with highest dermatitis grade, with the following mean increases in Skindex scores: 24 points (grade 1 dermatitis), 38 points (grade 2 dermatitis), and 53 points (grade 3 dermatitis)
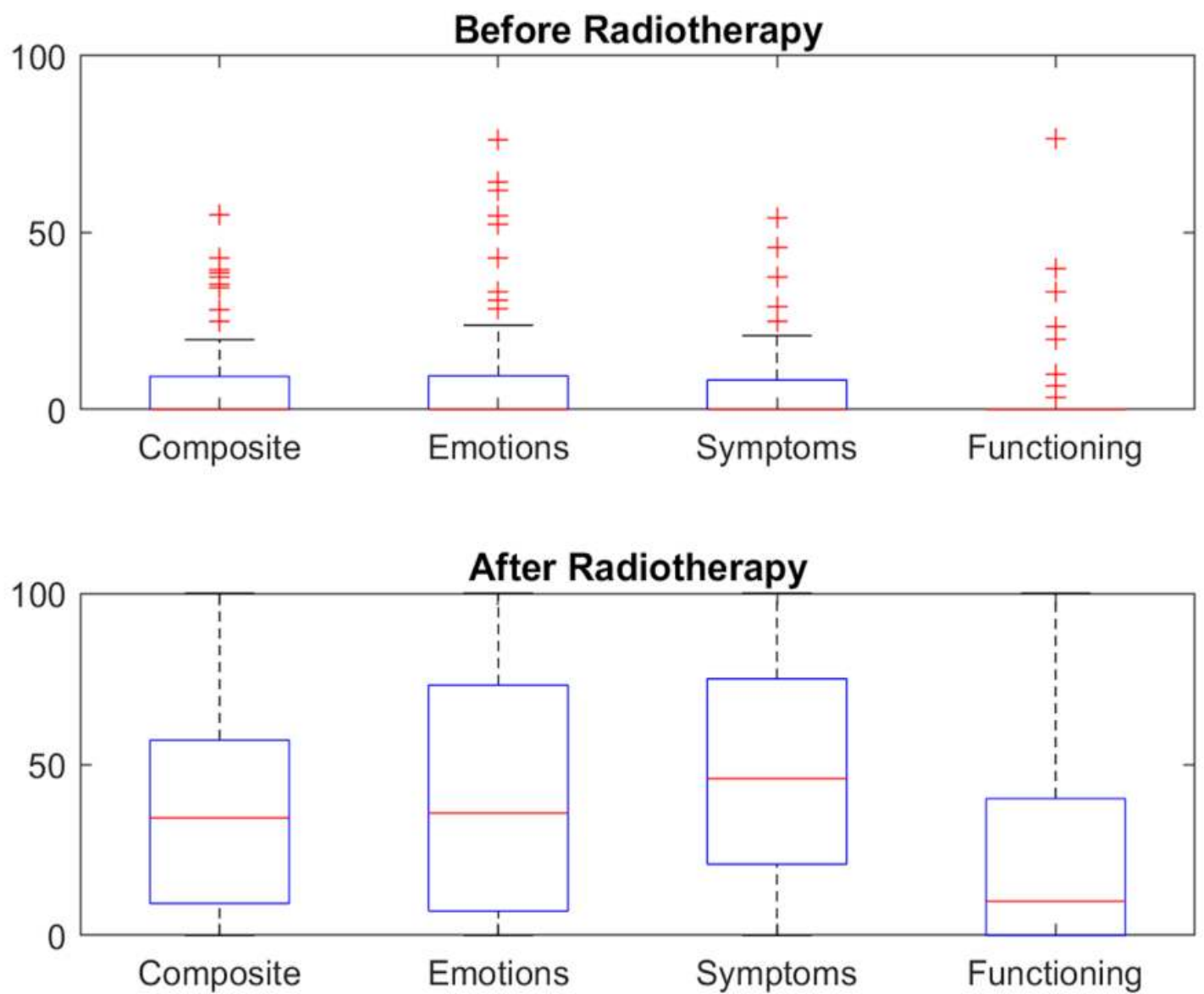

Figure 1. Median Skindex-16 scores, including composite and individual quality-of-life domains (emotions, symptoms, and functioning) for all patients pre- and post- RT

\section{Conclusion:}

- Cancer patients can experience a broad range of adverse dermatologic events during RT

- The CTCAE recommends evaluation of RD based on severity of erythema and desquamation, which excludes other domains that may be affected during RT such as emotional well being, functional status, and other common physical symptoms such as hyperpigmentation, skin dryness, and pain

- Evaluation using the Skindex-16 revealed that the severity of RD is significantly associated with a negative impact on QoL

- Increased awareness and focus on RD prevention may improve patient-reported outcomes

- Supportive interventions may need to address the emotional, functional, and symptomatic effects caused by radiotherapy-induced skin toxicity 